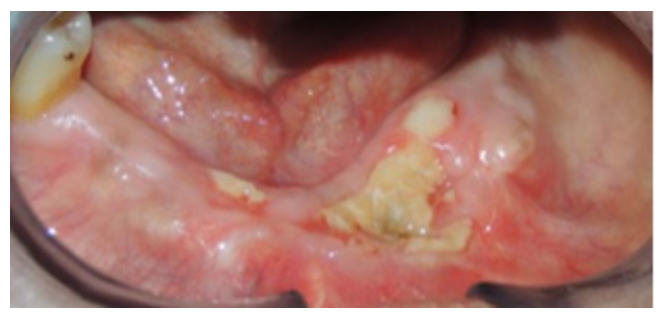

\title{
Management of Medication-Related Osteonecrosis of the Jaws: a monoinstitutional experience
}

\author{
Stefano Franchi ${ }^{1}$, Camilla Dosio ${ }^{1}$, Andrea Pezzana ${ }^{2}$, matteo brucoli ${ }^{1}$, Paolo Boffano ${ }^{3}$, Francesca Neirotti ${ }^{1}$, Anna Maria \\ Nora Mazzer ${ }^{1}$, Mila Bonaso ${ }^{4}$, annathea accornero ${ }^{1}$ \\ 1 University of Eastern Piedmont \\ 2 Azienda Ospedaliero Universitaria Maggiore della Carità \\ 3 ASL VC \\ 4 University of Turin
}

Funding: The author(s) received no specific funding for this work.

Potential competing interests: The author(s) declared that no potential competing interests exist.

\section{Abstract}

The aim of this communication is to present our protocol of management and treatment and to discuss our experience in the management of MRONJ patient at the Division of Maxillofacial Surgery at Novara Hospital in the last twelve years. A revision of all patients affected by MRONJ that were diagnosed between January 2009 and December 2020 was performed. On the whole, 109 patients with a mean age of 70 years were included: 75 patients had mandibular ONJ, whereas 27 maxillary ONJ and the remaining 7 patients mandibular and maxillary ONJ. 54\% of patients was classified as Stage 1, 30\% as Stage 2, and $16 \%$ as Stage 3. In 87 cases, patients underwent, in addition to medical therapy, surgical interventions of curettage, sequestrectomy, or bone resection; the remaining 22 patients were just administered medical therapy.

Advanced Stage MRONJ can be efficiently managed by surgery combined with medical therapy.

Background: Medication Related Osteonecrosis of the Jaw (MRONJ) is an established side effect of Bone Modifying Agents (BMA) such as bisphosphonates (BP) and Denosumab (DB). These drugs are used in antiresorptive therapy in patients with skeletal metastasis, emathological diseases like myeloma, or diseases of bone metabolism such as osteoporosis. Cases of MRONJ have also been reported in patients undergoing treatment with target therapy agents such as bevacizumab, sutinib and everolimus, either alone or in association with BMA. Currently (2014), MRONJ is defined as an area of exposed bone in the maxillofacial region that did not heal within 8 weeks after identification in a patients who 
was receiving or had been exposed to a antiresorptive or antiangiogenic therapy and had not had radiation therapy to the craniofacial region. The definition itself is currently object of discussion because of the evidence of cases without bone exposure. ${ }^{[1]}$

While incidence of MRONJ is yet undetermined, it was possible to estimate its frequency related to cancer and myeloma treatment, with data varying from 2 to 11.6/million/year of raw incidence in limited investigated populations and in different periods. [2]

The treatment of MRONJ often requires a combination of a more conservative approach (antimicrobial and antibiotic therapy) with surgical strategies including local debridement, osteoplasty, and marginal or segmental resection. ${ }^{[3]}$ We report data and clinical experience of the Oral and Maxillofacial Surgery Unit of the University Hospital of Novara "Ospedale Maggiore della Carità" in the management of MRONJ between January 2008 and December 2020.

Materials and Methods: Patients with MRONJ referred to the clinic undergo detailed collection of their medical history and thorough clinical and radiological (Orthopanoramic radiograph and CT scans) examination. We collected cases according to the AAOMS definition of MRONJ (bone exposure, no history of radiation therapy) as well as cases without frank bone exposure according to SIPMO-SICMF clinical and radiological criteria (Bedogni 2012).

The following data were collected:

- Age

- Gender

- Medical history. Patients were classified by pathology treated with antiresorptive drugs (oncological - emathological osteoporosis - other)

- Site of MRONJ: mandible, maxillary or multiple

- Stage of MRONJ according to SICMF-SIPMO criteria

- Treatment: non-surgical / minor surgery/ resective surgery

- Response to treatment: healing / relapse

Based on clinical evaluation (severity of MRONJ signs and symptoms, general conditions of the patients, comorbidities) and radiological staging by Computer Tomography, patients were proposed the following treatment options: 1) Nonsurgical treatment, with antimicrobial therapy (Chlorexidine $0.20 \%$ mouthwash rinses) in combination with broad spectrum antibiotic treatment. 2) Non-aggressive surgical treatment: mucosal and bone debridement, removal of spontaneous bone sequestra. 3) Radical resection of necrotic bone.

Results: Between January 2008 and December 2020, 109 patients (41 male, 68 female) with MRONJ were referred to our division of Oral and Maxillo-facial Surgery; median age of the patients was 70 years old (ranging between 45 and 93 years old). Of these, 61 (56,0\%) developed MRONJ after treatment for oncological disease (breast cancer: 33 cases; prostate cancer: 15 cases; lung cancer: 7 cases; kidney cancer: 3 cases; bladder cancer: 2 cases; colorectal cancer: 1 case), 28 $(25,7 \%)$ for haematological disease (multiple myeloma: 25 cases; Non-Hodgking Limphoma: 3 cases), and 20 (18,3\%) for osteoporosis. 
Most of the haematological patients (25 out on $28,89.3 \%$ ) had been treated with intravenous Zoledronate exclusively; the majority of oncological patients (40 out on $61,65,6 \%$ ) had also received Zoledronate alone (36 patients) or as the sequence Zoledronate-Denosumab (4 patients). Conversely, most of the osteoporotic patients (11 out on 20, 55\%) had been treated with oral Alendronate. Of the patients that received treatment with Denosumab (22, representing the 20,2 of the total), 14 oncological patients had been treated with DB alone; conversely, 6 oncological patients, 1 patient with myeloma and 1 patient with osteoporosis had received DB after a previous treatment with Zoledronate. In all cases, treatment with BPs or DB was discontinued after diagnosis of MRONJ.

Stages of MRONJ at presentation, according to SICMF-SIPMO staging system, were the following: Stage 1: 59 cases (54\%). Stage 2: 33 cases (30\%). Stage 3: 17 (16\%) cases.

In most cases, it was possible to identify the most probable cause of MRONJ in recent tooth extraction (40 cases, 36,7\%), dental disease (21 cases, 19,3\%), implants (6 cases, 5,5\%) or defective dentures (2 cases, 1,8\%); however, 39 cases $(35,8 \%)$ were spontaneous. however in one case of spontaneous multiple-site MRONJ, which was treated with major surgical resection, histological examination revealed simultaneous osteonecrosis and localization of myeloma cells both in the mandible and in the maxilla.

To all patients presenting MRONJ we prescribed antimicrobial therapy and antibiotic therapy, ether as an exlusive treatment or in preparation to the surgical treatment.

Of the 22 patients who received only the conservative treatment,

in 5 cases the patient did not undergo the programmed surgery because of the spontaneous sequestration of the necrotic bone. 6 patients, who could not undergo a surgical intervention, deceased in the immediate months following the diagnosis of MRONJ; 7 patients did not show improvement, while 4 patients achieved complete healing.

Surgical treatment was carried out for the majority of patients: 3 patients deceased within 6 month from the intervention, and 2 patients were lost to follow up. 57 patients received minor surgical treatment in the office under local anesthesia, while 25 underwent radical surgery under general anesthesia. We recorded relapse of bone exposure in 17 patients after minor surgery and 9 after major surgery; part of this patients (11 out on 26) achieved complete healing after a subsequent non-surgical treatment during follow-up, while the rest needed further surgery (9 patients), could not achieve healing (6 patients).

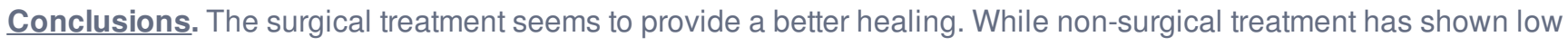
effectiveness, a minor surgical approach was sufficient for the treatment of most patients. In cases presenting with bone exposure after surgical treatment, some patients reach complete closure after a longer period, and non-surgical treatment may help in reaching a complete healing. 


\section{References}

1. 'A Bedogni, V Fusco, A Agrillo, G Campisi. (2012). Learning from experience. Proposal of a refined definition and staging system for bisphosphonate-related osteonecrosis of the jaw (BRONJ). Oral Diseases, vol. vol. 18 (6), 621-623.

2. ' V. Fusco, M. Cabras, F. Erovigni, A. Dell'Acqua, et al.. (2020). A multicenter observational study on MedicationRelated Osteonecrosis of the Jaw (MRONJ) in advanced cancer and myeloma patients of a cancer network in NorthWestern Italy.. Med Oral.

3. 'Campisi G, Bedogni A, Fusco V. (2020). Raccomandazioni clinico-terapeutiche sull'osteonecrosi delle ossa mascellari (ONJ) farmaco-relata e sua prevenzione.. 